\section{Cahiers de Narratologie}

Analyse et théorie narratives

$19 \mid 2010$

Images composites, arts pluriels

\title{
«L'Antiphilosophie du futurisme. Propagande, idéologie et concepts dans les manifestes de l'avant-garde italienne (1909-1944) » de Serge Milan
}

Patrick Marcolini

\section{CpenEdition}

Journals

Édition électronique

URL : http://journals.openedition.org/narratologie/6230

DOI : $10.4000 /$ narratologie.6230

ISSN : 1765-307X

Éditeur

LIRCES

Référence électronique

Patrick Marcolini, « "L'Antiphilosophie du futurisme. Propagande, idéologie et concepts dans les manifestes de l'avant-garde italienne (1909-1944) » de Serge Milan », Cahiers de Narratologie [En ligne], 19 | 2010, mis en ligne le 22 décembre 2010, consulté le 19 avril 2019. URL : http:// journals.openedition.org/narratologie/6230; DOI : 10.4000/narratologie.6230

Ce document a été généré automatiquement le 19 avril 2019

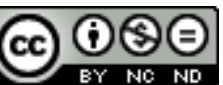

Cahiers de Narratologie - Analyse et théorie narratives est mis à disposition selon les termes de la licence Creative Commons Attribution - Pas d'Utilisation Commerciale - Pas de Modification 4.0 International. 


\title{
"L'Antiphilosophie du futurisme. Propagande, idéologie et concepts dans les manifestes de l'avant-garde italienne (1909-1944) » de Serge Milan
}

\author{
Patrick Marcolini
}

\section{RÉFÉRENCE}

Lausanne, Editions L'Âge d'Homme,2010

1 L'Antiphilosophie du futurisme est un ouvrage d'importance dans l'histoire des recherches sur l'avant-garde futuriste italienne, tant par l'ampleur du matériau traité (plus de deux cents manifestes soigneusement sélectionnés) que par les nouvelles perspectives qu'il ouvre sur le sujet: la production idéologique de ce mouvement, ses origines intellectuelles, les lignes conceptuelles à partir desquelles il a formulé son programme en art, mais aussi son impact en termes de propagande.

2 L'ouvrage se présente comme un fort volume augmenté de photographies et reproductions, ainsi que d'un choix de manifestes futuristes dont certains sont traduits en français pour la première fois. Il impressionne par sa capacité à brasser un corpus massif et hétérogène (regroupant des écrits parfois fort différents publiés de 1909 à 1944), et par la sensibilité simultanément littéraire et philosophique qui guide l'analyse de ces textes, tout au long du propos. En effet, nous avons là une étude à la lecture de laquelle le philosophe ou l'historien des idées trouvera tout autant son compte que le spécialiste en littérature et civilisation italiennes.

De ce point de vue, le terme d'antiphilosophie ici mis en avant ne doit pas surprendre. Emprunté aux futuristes eux-mêmes, il rend compte de leur volonté de ne pas formaliser trop abstraitement les principes du mouvement, par méfiance vis-à-vis de tout 
intellectualisme desséchant, mais aussi leur ambition de produire une véritable conflagration dans la culture italienne et occidentale, en faisant de l'art une puissance de rupture vis-à-vis de toutes les pensées héritées, instituées. Mais l'antifilosofia révèle également de la part de Marinetti et de ses comparses un sens certain de la publicité, du scandale et de la polémique, qui fait de chaque mot un obus, de chaque thèse une formule-choc, de chaque esquisse de raisonnement une petite machine de guerre à conquérir les esprits - en somme tout l'inverse de ce que prétendent faire (officiellement) les philosophes authentiques. Et pourtant, comme y insiste Serge Milan, le futurisme en tant qu'idéologie recouvre bien un discours sur le monde, un discours sur l'homme et un discours sur l'art, qui, pour être normatifs, n'en sont pas moins révélateurs d'une philosophie sous-jacente.

En effet, Serge Milan ne se borne pas à souligner les inspirations que les futuristes ont pu trouver chez des philosophes comme Nietzsche, Bergson ou Sorel - dont les influences sur le mouvement, souvent explicites, avaient déjà été remarquées par d'autres interprètes. A travers la lecture des manifestes, il entreprend un travail de reconstitution de la doctrine futuriste jusqu'ici éparse, écartant volontairement les références étrangères au mouvement pour mieux l'éclairer de l'intérieur, selon ses lignes de force et sa logique propre. En un sens, plus qu'à un travail herméneutique, l'auteur s'est livré ici à une sorte de travail maïeutique sur les textes: les faire accoucher de la philosophie dont ils sont gros, de la cosmologie, de la poétique et de l'anthropologie dont ils sont porteurs - puisque telles sont les trois grandes catégories qu'on voit déployées au cours de l'analyse, et qui lui servent de charpente.

5 Sur le plan cosmologique, Serge Milan insiste sur ce qu'il nomme l'ontologie dogmatique du futurisme: son refus des essences fixes et inaccessibles à l'écoulement du temps, en d'autres termes son mobilisme ou son héraclitéisme; la valorisation du hasard et du risque liés à cette instabilité constitutive de l'univers, à cette cinétique perpétuelle des phénomènes; et enfin, l'artificialisme propre au futurisme, qui le conduit à vouloir reconstruire intégralement le monde sur la base de l'inventivité des arts, sciences et techniques mêlés.

6 La poétique du mouvement futuriste n'est finalement que la traduction de cette cosmologie sur le plan des formules artistiques. La valorisation du mouvement et de la nouveauté s'y traduit par le refus du passéisme et de la nostalgie, par lesquels le futurisme s'affirme comme un mouvement intrinsèquement anti-romantique. Le principe de la tabula rasa vis-à-vis de toute tradition, de tout héritage historique, est en ce sens revendiqué comme facteur d'émancipation pour la création artistique. C'est d'ailleurs à ce principe que s'arrime en grande partie le projet d'une unification entre l'art et la science proposé par plusieurs futuristes, dans la mesure où la science, elle aussi, procède en permanence à un bouleversement général des données acquises, au moyen d'expériences permettant de faire advenir du nouveau - ce Nouveau auquel les futuristes vont conférer une valeur absolue. Mais l'aspect le plus intéressant de cette poétique futuriste telle que Serge Milan nous la donne à comprendre est sans aucun doute le concept de «nouvelle sensibilité ", récurrent dans les textes du mouvement, et dont la signification reste parfois énigmatique, quasiment allusive: une sensibilité émergeant des soubresauts de la vie urbaine et technicisée, surdéterminée par les transformations de ces organes de la vie collective que sont les médias de masse, et dont les ressources doivent être artistiquement exploitées, multipliées et potentialisées. 
7 C'est sans doute l'anthropologie du futurisme qui donne la clé de ce concept de « nouvelle sensibilité ». Serge Milan y montre en effet comment le mouvement a rêvé de créer un homme nouveau sur la base des sciences et des techniques modernes-s'approchant parfois de ce qui s'élabore aujourd'hui du côté de la cybernétique ou des biotechnologies. Ce fantasme qui habite un bon nombre de productions futuristes s'accompagne d'une réflexion sur la constitution d'une nouvelle table des valeurs adaptée aux réalités du XX ème siècle - c'est-à-dire en fait à cette "guerre civile mondiale " dont le déroulement recouvre presque exactement la durée de vie de l'avant-garde emmenée par Marinetti. Force, courage, héroïsme, ivresse, surpassement de la tristesse et de la souffrance dans le rire aux éclats: l'ethos futuriste est un ethos foncièrement guerrier, lui aussi orienté par la recherche d'un au-delà de l'humain autour duquel tournent toutes les utopies futuristes, que leur traduction soit spéculative (voire quasiment religieuse) ou plus directement politique (à travers la brève expérience du Parti politique futuriste dans les années précédant la marche sur Rome).

8 L'originalité de l'ouvrage de Serge Milan est donc d'avoir intégré cette reconstitution méticuleuse, passionnée, des principaux idéologèmes futuristes, au sein d'une tripartition empruntée à la philosophie classique, dont la progression est d'ordre logique: de la vision du monde partagée par les futuristes à sa réorganisation utopique, en passant par la conception de l'art qui en est le centre agissant. L'originalité ainsi que les conclusions de cette démarche invitent à la réitérer, mutatis mutandis, à partir d'autres points d'observation, comme celui d'une histoire des idées ancrée dans l'histoire matérielle des sociétés occidentales. D'un tel point de vue, le futurisme peut être interprété comme une expression culturelle des bouleversements introduits par la seconde révolution industrielle dans les sociétés humaines, en ce qu'il partage non seulement les idéaux scientifiques liés à cette révolution (méthodologie de la tabula rasa et de l'expérimentation, etc.), mais aussi les rêves qui en sont issus (fusion de l'homme avec la machine, fantasme d'immortalité par la technologie, préfiguration de la cybernétique), tout en faisant l'apologie des formes de vie et de sensibilité qui en sont les conséquences directes (civilisation des grandes métropoles, influence des mass media, habituation au choc, à la vitesse, etc.). Si l'on part de ce constat, le futurisme serait alors susceptible d'une analyse à rebours, ses principes métaphysiques apparaissant comme une extrapolation idéologique de son programme artistique, celui-ci étant lui-même déterminé par son anthropologie originaire-qui reflète en réalité la mutation anthropologique à l'œuvre au sein des sociétés travaillées par la seconde révolution industrielle.

Quoi qu'il en soit, ceci n'est qu'un exemple parmi d'autres, suggérant dans l'histoire des idées et des faits matériels un prolongement possible des analyses effectuées par Serge Milan à partir d'un positionnement littéraire et philosophique. Bien d'autres approches trouveraient en effet à se satisfaire des conclusions apportées par cette « antiphilosophie du futurisme ", et c'est bien tout le mérite d'une telle étude que d'ouvrir à ce point le champ des interprétations et des hybridations méthodologiques. 